\title{
Cytotoxicity of Bacterial Metabolic Products, including Listeriolysin 0 , on Leukocyte Targets
}

\author{
R. Stachowiak, ${ }^{1}$ M. Lyzniak, ${ }^{2}$ B. K. Budziszewska, ${ }^{3}$ K. Roeske, ${ }^{1}$ J. Bielecki, ${ }^{1}$ \\ G. Hoser, ${ }^{2}$ and J. Kawiak ${ }^{2}$ \\ ${ }^{1}$ Department of Applied Microbiology, University of Warsaw, 02-096 Warsaw, Poland \\ ${ }^{2}$ Department of Clinical Cytology, Medical Centre of Postgraduate Education, 01-813 Warsaw, Poland \\ ${ }^{3}$ Department of Haematology, Institute of Haematology and Transfusion Medicine, 02-776 Warsaw, Poland
}

Correspondence should be addressed to J. Kawiak, jkawiak@cmkp.edu.pl

Received 22 May 2012; Accepted 24 July 2012

Academic Editor: Frederick D. Quinn

Copyright (c) 2012 R. Stachowiak et al. This is an open access article distributed under the Creative Commons Attribution License, which permits unrestricted use, distribution, and reproduction in any medium, provided the original work is properly cited.

\begin{abstract}
Bacterial toxins can exhibit anticancer activities. Here we investigated the anticancer effects of the listeriolysin O toxin produced by Listeria monocytogenes. We found that supernatants of Listeria monocytogenes strains (wild type, 1189, and 1190) were cytotoxic to the Jurkat cell line and human peripheral blood mononuclear cells (PBMC) in a concentration-dependent manner. The supernatant of strain 1044, not producing listeriolysin O, was inactive. The supernatants of Listeria strains were also cytotoxic toward B cells of chronic leukemia patients, with no significant differences in activities between strains. We also tested supernatants of Bacillus subtilis strains BR1-90, BR1-S, and BR1-89 producing listeriolysin O. BR1-S and BR1-89 were cytotoxic to PBMC and to Jurkat cells, the latter being more sensitive to the supernatants. BR1-90 was not hemolytic or cytotoxic to PBMC, but was cytotoxic to Jurkat cells in the concentration range of 10-30\%, suggesting that listeriolysin O is selectively effective against $\mathrm{T}$ cells. Overall, the response of human peripheral blood mononuclear and human leukemia cell lines to bacteria supernatants containing listeriolysin $\mathrm{O}$ depended on the bacteria strain, target cell type, and supernatant concentration.
\end{abstract}

\section{Introduction}

The anticancer role of bacteria was recognized over a hundred years ago, when the American physician William Coley began the first well-documented use of bacteria and their toxins to treat end-stage cancers. He developed a safe vaccine composed of killed bacteria species [1], which was used to successfully treat sarcomas, carcinomas, lymphomas, and melanomas [2].

Most pathogenic bacteria produce toxins; these important pathogenic factors are generally proteins with enzymatic activity that can be classified into several groups. The first class includes hydrolytic enzymes, such as phospholipases, proteases, metalloproteases, glycosidases, deaminases, and deoxyribonucleases, which disrupt target molecules. Another class interferes with host metabolism modifies metabolic components; this group includes transferases, such as glucosyl transferases and ADP-ribosylating toxins, or lyases like adenylate cyclase [3]. A third group of toxins, the cytolysins, acts on the cytoplasmic membranes, without enzymatic activity or intracellular penetration; this group includes receptor-targeted toxins and membrane-damaging toxins, which are produced by numerous Gram-positive and Gram-negative bacteria. Cytolysins may combine cytolytic abilities with enzymatic activity, and some phospholipases can hydrolyze lipids in the cell membrane together with membrane-lysing activity.

Pore-forming cytolysins are membrane-damaging toxins without enzymatic activity. These highly specialized proteins damage target cells by inserting hydrophobic regions into the cellular membrane phospholipid bilayer [3]. The largest and most homogenous group of pore-forming cytolysins are the cholesterol-dependent cytolysins (CDCs). The CDCs are exclusively produced by Gram-positive pathogens, and share similar amino acid sequences and biochemical properties [4]. Like other toxins, CDCs are usually secreted by a bacterial cell such that their activity can be directly exerted on eukaryotic cells. Efficient secretion machinery is typical for 
most pathogenic bacteria and nonpathogenic bacteria that are able to substantially modify their surroundings.

Listeria monocytogenes is an intracellular pathogen that efficiently secretes pathogenicity determinants, including three toxins. One of its toxins is listeriolysin O (LLO), a CDC that is crucial for pathogen intracellular survival $[5,6]$. The two other toxins are phospholipases, designated PlcA and $\mathrm{PlcB}$, which both enhance LLO cytolytic activity [7] and $L$. monocytogenes virulence $[8,9]$. Bacillus subtilis is a nonpathogenic, model Gram-positive bacterium; it is well known for its enormous secretion machinery, and is widely used in both research and industry for efficient protein production [10-12]. Listeria and Bacillus secretion genes are closely related and share similar secretion apparatus. The main secretory pathway of both organisms is SecA, which is used to transport LLO [13]. These similarities enable highly effective expression and secretion of listeriolysin $O$ in $B$. subtilis [14]. We previously used LLO-secreting B. subtilis in in vitro experiments with the human Jurkat cell line $[15,16]$. Therefore, in the present study, we used both B. subtilis and L. monocytogenes for cytolysin production.

Here we present the results of in vitro experiments of the hemolytic and cytotoxic activities of bacteria culture supernatants on human peripheral blood leukocytes, both normal and leukemia. We used L. monocytogenes strains that synthesized LLO and mutant strains that produced variants of this toxin, along with engineered $B$. subtilis strains harboring the wild-type or mutated hlyA gene. The use of LLO-producing B. subtilis strains is an attractive idea because it allows the study of this toxin in the isolation from other pathogenesis factors [15]. This technique also potentiates the practical use of such toxins, since the use of B. subtilis is safer than use of the original pathogenic host.

\section{Materials and Methods}

2.1. Bacterial Strains and Growth Conditions. The bacterial strains used in this study are listed in Table 1.

Bacteria were grown in LB broth (Sterbios, Warsaw, Poland) or brain heart infusion broth (BHI; Beckton Dickinson, Difco, New Jersey, USA) at $37^{\circ} \mathrm{C}$ with shaking at $120 \mathrm{rpm}$. L. monocytogenes strains were grown overnight in $\mathrm{BHI}$, then diluted in BHI broth containing 1\% Amberlite (Merck) to enhance cytotoxin production, and cultured until reaching early stationery phase. $B$. subtilis overnight cultures were grown in LB and then diluted and grown in BHI until OD 1.5 was reached. To induce LLO synthesis, isopropyl- $\beta$ D-thiogalactoside (IPTG) was added to a final concentration of $0.5 \mathrm{mM}$. Samples were taken for assays, and all strains had similar OD values. Overall proteins extracts were analyzed by PAGE to check LLO content. All stock bacteria cultures were stored at $-20^{\circ} \mathrm{C}$ as suspensions of cells in $20 \%$ glycerol.

2.2. Construction of B. subtilis Mutant Strains. L. monocytogenes strains 1189 and 1190 [18] were grown overnight in BHI, washed with PBS and pretreated with lysozyme before DNA isolation with chromosomal DNA isolation kit (A\&A Biotechnology). Chromosomal DNA was matrix for PCR (Qiagen PCR kit) with following primers: left: 5' GCTCTAGAAGAGAGGGGTGGCAAACGGT 3' (XbaI site underlined) and right: 5' GCGGTCGACGGTACCTTTCGTGTGTGTTAAGCGGT 3' (KpnI and SalI sites underlined). PCR products and destination vector pAG58 were digested with XbaI and SalI enzymes (Fermentas) and purified with Clean-up kit (A\&A Biotechnology). Vector and insert DNA were ligated (Fermentas) and transformed to E. coli for construct verification. Selected clones were subject for plasmid isolation and recombinant plasmids were transformed into B. subtilis MB4 strain [21] to produce BR1-89 and BR1-90 (harboring hly gene from L. monocytogenes strains 1189 and 1190, resp.).

2.3. Eukaryotic Cell Isolation and Growth Conditions. The following target Eukaryotic cells were used: sheep red blood cells (SRBC; Biomed, Warsaw, Poland), human acute T cell leukemia cell line (Jurkat; ATCC TIB 152), peripheral blood mononuclear cells (PBMC) from healthy humans, and PBMC from B-cell chronic lymphocytic leukemia (B-CLL) patients.

The Jurkat cells were grown in RPMI 1640 (Gibco) culture medium containing 10\% inactivated calf serum (NCS) with $100 \mathrm{U}$ penicillin and $0.1 \mathrm{mg}$ streptomycin per $\mathrm{mL}$, or in DMEM medium (Sigma) with 10\% inactivated calf serum (NCS). NCS contained $150 \mathrm{mg} / \mathrm{dL}$ cholesterol.

Human peripheral blood mononuclear cells (PBMC) were isolated and cultured in vitro for 2-7 days before cytotoxicity experiments. For the in vitro peripheral blood experiments, patients gave their consent, and the procedure was accepted by the Bioethical Commission at the Medical Center of Postgraduate Education, Warsaw (Feb 27, 2008). PBMC isolation from both healthy subjects and B-CLL patients was performed by overlaying $10 \mathrm{~mL}$ of peripheral blood diluted with PBS $(1: 1)$ over $5 \mathrm{~mL}$ of Histopaque 1077 (Sigma) that was prepared in a test tube, followed by centrifugation for $30 \mathrm{~min}$ at $540 \times \mathrm{g}$. The interlayer of PBMC containing lymphocytes and monocytes was separated, diluted with $5 \mathrm{~mL}$ of culture medium, and centrifuged $5 \mathrm{~min}$ at $200 \times \mathrm{g}$. Then the washed cells were suspended in new culture medium and counted using the Burker camera. The concentration of the cell suspension was adjusted to a final value of $2 \times 10^{6} / \mathrm{mL}$ before culture. The cultures were established in Falcon vessels (dishes) or in 6-well flat-bottom plates, in RPMI 1640 with $10 \%$ NCS and antibiotics; half of the medium volume was changed twice per week.

2.4. Supernatants of Bacteria Culture Medium. Bacterial cultures were centrifuged for $5 \mathrm{~min}$ at $4300 \times \mathrm{g}$. The culture medium supernatant was passed through a $0.22 \mu \mathrm{m}$ filter (Millipore), then frozen at $-20^{\circ} \mathrm{C}$ in the presence of $5 \mathrm{mM}$ cysteine and $10 \%$ glycerol ( $\mathrm{pH} 6$ ), and stored for later use in the activity assays.

2.5. Hemolytic Activity Assay. Hemolytic activity was assayed on sheep erythrocytes (SRBC). The erythrocytes were washed three times with PBS, and suspended to a final concentration of $10 \%$ in PBS ( $\mathrm{pH}$ 7.4). The supernatant 
TABLE 1: List of bacterial strains.

\begin{tabular}{|c|c|c|}
\hline Strain & Characteristics & References \\
\hline L. monocytogenes $10403 \mathrm{~S}$ & Wild type & [17] \\
\hline L. monocytogenes 1189 & $\begin{array}{l}10403 S \text { strain harbouring point mutation in hly gene resulting in Cys484Ala } \\
\text { substitution in LLO protein. }\end{array}$ & {$[18]$} \\
\hline L. monocytogenes 1190 & $\begin{array}{l}10403 S \text { strain harbouring point mutation in hly gene resulting in Cys } 484 \text { Ser } \\
\text { substitution in LLO protein. }\end{array}$ & {$[18]$} \\
\hline L. monocytogenes 1044 & Nonhemolytic derivative of $10403 S$. Insertional mutant in $h l y A$ gene. & [19] \\
\hline L. monocytogenes MAC & $\begin{array}{l}\text { Mackaness strain, hypervirulent isolate harbouring natural } \operatorname{pr} f A^{*} \text { mutation, } \\
\text { overproducing determinants of pathogenesis. }\end{array}$ & {$[20]$} \\
\hline B. subtilis MB4 & $\begin{array}{l}\text { Asporogenic derivative of strain ZB307 containing a } \\
\text { spoIIE::Tn9I7fQHU181::1ac55 insertion }\end{array}$ & {$[21,22]$} \\
\hline B. subtilis BR1-S & MB4/pAG58/hly & {$[16]$} \\
\hline B. subtilis BR1-89 & MB4/pAG58/hly $y^{C 484 A}$ & This work \\
\hline B. subtilis BR1-90 & MB4/pAG58/hly ${ }^{C 484 S}$ & This work \\
\hline
\end{tabular}

was diluted by adding $20 \mu \mathrm{L}$ to a final volume of $1 \mathrm{~mL}$ $1 \%$ erythrocyte suspension. The prepared solution was incubated for $30 \mathrm{~min}$ at $37^{\circ} \mathrm{C}$, and then centrifuged for $3 \mathrm{~min}$ at $150 \times \mathrm{g}$. The released hemoglobin was measured spectrophotometrically at $410 \mathrm{~nm}$. Hemolytic units (HU) were calculated after setting $0 \mathrm{HU}$ as the activity of the negative control, and $100 \mathrm{HU}$ as total hemolysis as determined in an erythrocyte sample lysed with $0.01 \%$ SDS.

2.6. Western Blot Analysis of Supernatants. B. subtilis supernatants derived proteins and molecular weight marker (PageRuler Plus Prestained Protein Ladder, Fermentas) were separated by SDS-PAGE and were transferred to nitrocellulose membrane by using the BIO-RAD Trans-Blot system according to the manufacturer's protocol. The membrane was blocked in 5\% skimmed milk in Tris-buffered saline (TBS) pH 7.6. Primary rabbit polyclonal anti-LLO antibody (Abcam) and secondary goat polyclonal antibody (Abcam) conjugated with alkaline phosphatase were used at 1/1500 and $1 / 10000$ dilutions, respectively. The membrane was developed using Calbiochem NBT/BCIP reagent.

2.7. Fluorescence Microscopy Cytotoxicity Assay. Bacterial supernatant activity on Jurkat cells was observed using the florescence microscope. Supernatants were obtained from $L$. monocytogenes strains producing LLO (MAC, 1190, 1189, and 10403S), as well as from a strain not producing LLO (1044) as the negative control. B. subtilis strains producing LLO were used (BR1-S, BR1-89 and BR1-90) and parental strain (MB4) as the negative control. We mixed $900 \mu \mathrm{L}$ of the Jurkat cell suspension in a well with $100 \mu \mathrm{L}$ of bacterial supernatant, and incubated the mixture for $60 \mathrm{~min}$ (L. monocytogenes supernatants) or $30 \mathrm{~min}$ (B. subtilis supernatants) at room temperature. The final cell concentration was $1 \times$ $10^{6} / \mathrm{mL}$. Next, $100 \mu \mathrm{L}$ of calcein-ethydine testing solution was applied for $30 \mathrm{~min}$. Green calcein fluorescence indicated living cells, while damaged cells were observed as the red fluorescence of ethydine binding to the cell nucleus. The cell preparation was photographed with the Olympus IX70 microscope in three independent fields of view $(760.9 \mu \mathrm{m}$ per $570.7 \mu \mathrm{m}$ ), and each experiment was repeated three times. Live (Green fluorescence) and dead (red fluorescence) cells were counted automatically using DP-Soft (analySIS) application.

2.8. Cytometric Assays. The activities of L. monocytogenes supernatants (strains MAC, 1190, 1189, 10403S, and 1044) were tested on Jurkat cells. We mixed $20 \mu \mathrm{L}$ of the $1 \times$ $10^{6} / \mathrm{mL}$ Jurkat cell suspension with $30 \mu \mathrm{L}$ of the bacterial supernatant, which was diluted with physiological salt solution (PSS) at different proportions such that the amounts of bacterial supernatant were $1,2,3,4,5,10,20,30,40$, and $50 \%$ of the total assay volume of $50 \mu \mathrm{L}$. The final cell concentration was $0.4 \times 10^{6} / \mathrm{mL}$ and the final concentration of FCS from the culture medium was $4 \%$ ( $6 \mathrm{mg}$ cholesterol/dL). The mixture was immediately incubated for $30 \mathrm{~min}$ at room temperature.

After incubation, $300 \mu \mathrm{L}$ of PSS and $1 \mu \mathrm{L}$ of propidium iodide (PI) solution $(0.1 \mathrm{mg} / \mathrm{mL}$ water) were added. Propidium iodide penetrates the cell membrane of damaged cells and binds to the cell nucleus, such that the percentage of living cells is indicated by the PI-unstained events in a fluorescence quadrant reading of FSC/PI fluorescence. We used a FACSCalibur cytometer to collect 5000 events from each sample, which were then analyzed using the Cell Quest application (Becton Dickinson). All experiments were repeated three times.

Similar analyses were performed with PBMC suspensions from normal peripheral blood cells and from cells of BCLL patients. The supernatants of $B$. subtilis strains with the hly gene (BR-1-89, BR-1-90, and BR-1-S) were also tested similarly by cytometry, and compared with the supernatant of wild-type strain B. subtilis MB4 and with the negative control BHI medium. 
2.9. Statistical Analysis. The cytometric results are presented as median values and percentile values, $\mathrm{P}_{25}$ and $\mathrm{P}_{75}$. Statistical analysis of cytometric experiments was performed using the global linear model (GLM) after logit transformation of values. Tukey's post hoc test was also used. Results of hemolytic tests and fluorescence microscopic measurements are presented as mean values $\pm \mathrm{SD}$. Analysis of variance was performed with ANOVA. Differences where $P$ was $<0.05$ were considered to be significant. The statistical application SAS 9.2 was used.

\section{Results}

3.1. Hemolytic Activity of Bacterial Strains. The cytolytic activities of bacterial supernatants were tested on SRBC. After spectrophotometric measurements, HU was calculated; the absorbance of the negative control was assumed to be 0 $\mathrm{HU}$ (spontaneous hemolysis), and the absorbance following lysis caused by $0.01 \%$ SDS was set as $100 \mathrm{HU}$. Activities of $L$. monocytogenes supernatants in neutral conditions ( $\mathrm{pH} 7.4$ ) are shown in Figure 1(a). The control nonhemolytic strain 1044 showed $0 \mathrm{HU}$. The highest activity was recorded for the MAC strain (74 HU). The remaining strains presented decreasing activities, from strain $10403 \mathrm{~S}$ to 1190 . The differences between the hemolytic activities of these strains were not statistically significant.

The results of hemolytic assays with supernatants of $B$. subtilis strains are presented in Figure 1(b). No hemolytic activity was observed with the control strain MB4 or with the BR1-S (-) strain grown without IPTG. The mean hemolytic activity of the supernatant of strain BR1-S (+) induced with IPTG was $54 \mathrm{HU}$. The highest activity was observed with the BR1-89 + IPTG supernatant on SRBC (87 HU). Strain BR1$90+$ IPTG showed minimal activity (5 HU). Western blot analysis confirmed the presence of a LLO $58-\mathrm{kDa}$ streak in B. subtilis supernatants (Figure 2). Other bands are result of nonspecific rabbit polyclonal anti-LLO antibody binding.

3.2. Cytotoxic Activity of Bacterial Supernatants on Jurkat Cells Determined by Fluorescence Microscopy. We assessed the cytotoxic effects of toxins produced by L. monocytogenes and B. subtilis strains on Jurkat cells. Eukaryotic cell viability was evaluated microscopically after staining with calcein AM and ethidium homodimer, and the cytotoxicity assay results are shown in Figure 3.

The strongest cytotoxic effect on Jurkat cells was recorded for L. monocytogenes MAC strain (35\% of dead cells). Cytotoxicity of 1189 and 1190 is at similar level (over 20\%) while the activity of L. monocytogenes wild-type (10403S) is surprisingly low. Overall, higher cytotoxicity levels were observed for L. monocytogenes strains than for B. subtilis. These results, however, are not directly comparable as in hemolytic test (Section 3.1) since different incubation time was used for each experiment. Actually, B. subtilis supernatants tend to be more active (Figure 1), possibly due to a higher cytotoxins content, therefore $30 \mathrm{~min}$ incubation of Jurkat cells with B. subtilis supernatants was sufficient while 60 min was necessary to observe significant cytotoxicity in case of L. monocytogenes supernatants. The highest cytotoxicity (over 10\% dead cells) among B. subtilis strains was observed following treatment with the supernatant of the B. subtilis BR1-89 strain producing listeriolysin $\mathrm{O}$ in which cysteine 484 was substituted by alanine. Significant cytotoxicity was also observed with the supernatant of the BR1-S strain producing unmodified toxin. Strains BR1-90 and MB4 displayed minimal cytotoxicity toward Jurkat cells.

3.3. Activity of L. Monocytogenes Supernatants Against Jurkat Cells or Peripheral Blood Mononuclear Cells; Cytometric Assays. Propidium iodide was used to evaluate cell survival; damaged cells fluoresced above the background level due to the fluorochrome binding to the DNA of apoptotic and necrotic cells. Table 2 presents the survival analysis of Jurkat cells treated with rising concentrations of supernatants of $L$. monocytogenes strains (1189, 1190, 10403S, and 1044).

Supernatants of all strains were active when compared with the supernatant of the negative control strain 1044 (GLM: $P<0.05$, Tukey: $P<0.05$ ), there were no statistically significant differences in cytotoxic activity between supernatants of the tested LLO-containing strains. Similar results were observed when examining the Jurkat cell line with a fluorescence microscope (Figure 3). In this experiment, additionally MAC strain supernatant activity was measured and it proved to be the highest (Tukey: $P<0.05$ )

The activities of the supernatants depended on their concentration (GLM: $P<0.05$ ). The cytotoxicity of the supernatants of Listeria strains 1189 and 1190 to Jurkat cells started at a concentration of $10 \%$, while the supernatant of the wild-type strain $10403 \mathrm{~S}$ was active already at a concentration of $3-4 \%$. These results were comparable to those of the hemolytic test, and are presented in Table 3.

Similar cytotoxic analyses were performed on the PBMC of healthy subjects (Table 3 ) and of B-cell chronic lymphocytic leukemia patients (Table 4). On PBMC of healthy subjects, the most active supernatant was that of the Listeria MAC strain; statistically significant differences were observed at supernatant concentrations of $10-50 \%$. We also observed somewhat different results with the supernatant of the 10403S strain as compared with 1189 and 1190; however, these differences were not significant. As observed in other analyses, the supernatant of the 1044 strain was inactive in the tested concentration range (Tables 3 and 4 ). There were no differences in the activities of supernatants of different Listeria strains on the PBMC of B-CLL patients (Tukey: $P>$ 0.05, GLM: $P<0.05$ ), as presented in Table 4 .

In summary, the Listeria monocytogenes supernatants of the wild-type strain and mutant strains 1189 and 1190 were active in concentrations of 10-20\%. The control strain 1044 supernatant was inactive at all tested concentrations. Statistically significant differences in the activities of supernatants of all tested strains were observed when compared with the activity of the control strain supernatant $(P<$ 0.05). The cytotoxic activities of the tested samples were significantly concentration dependent $(P<0.05)$ (Tables $2-$ 4). The supernatant of the MAC strain had a significantly different activity on PBMC from healthy subjects compared 


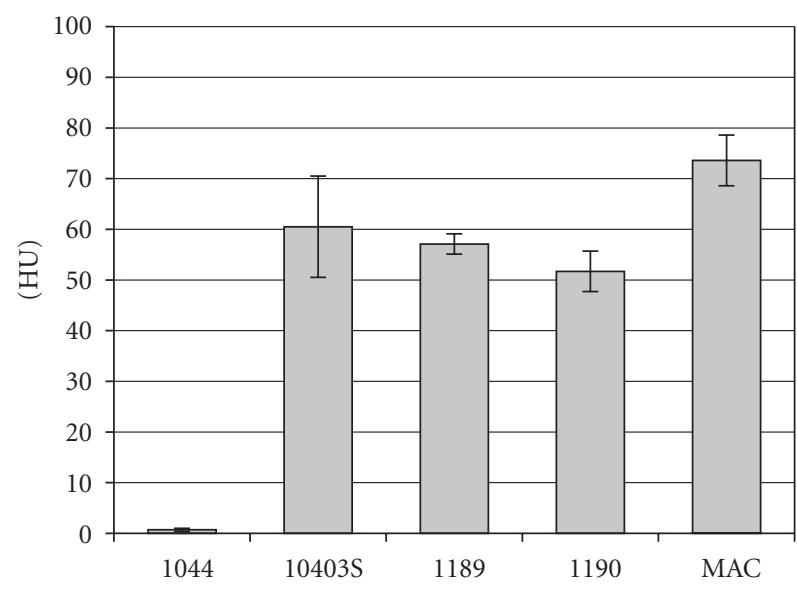

(a)

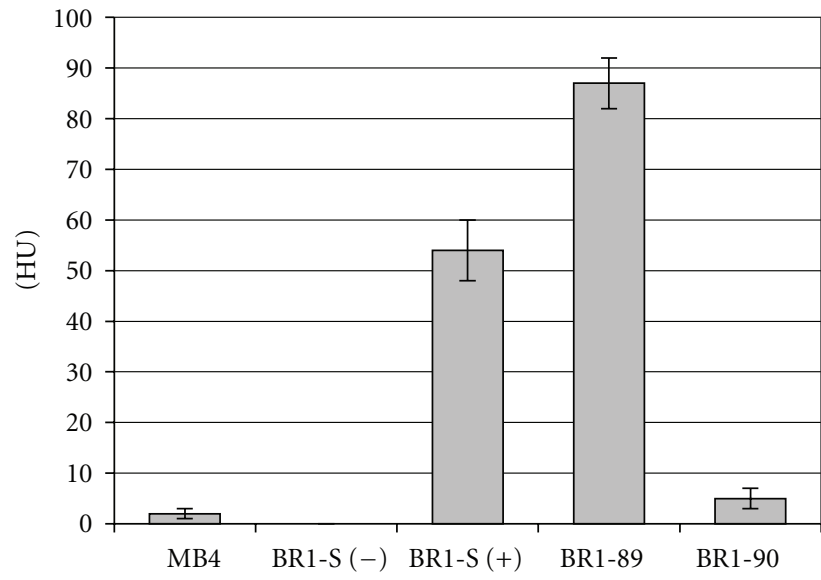

(b)

FIGURE 1: Hemolytic activity of L. monocytogenes (a) and B. subtilis (b) strains. Spectrophotometric measurements of the hemolytic activity of the bacterial supernatants of Listeria monocytogenes (a) and Bacillus subtilis (b) strains tested on sheep erythrocytes (SRBC). The negative control was $20 \mu \mathrm{L}$ of BHI medium, $2 \%$ of final sample volume, exactly as in the tested supernatants. Hemolysis of the negative control was defined as zero hemolytic units (HU). The positive control absorbance was set to $100 \mathrm{HU}$, defined as the hemolysis caused by $20 \mu \mathrm{L}$ of $0.5 \%$ SDS $(0.01 \%$ final concentration). BR1-S $(-)$ is B. subtilis strain grown without IPTG, BR1-S (+) the same strain induced with IPTG. Hemolytic activity of the bacterial strains was calculated according to the absorbance of controls. The average of three independent experiments $(n=3)$ is indicated with bars showing standard deviation.

TABLE 2: Survival of Jurkat cells treated with different concentrations of L. monocytogenes strain supernatants.

\begin{tabular}{|c|c|c|c|c|c|c|c|c|c|c|c|}
\hline \multirow{2}{*}{\multicolumn{2}{|c|}{ Strain }} & \multicolumn{10}{|c|}{ Supernatant concentrations } \\
\hline & & $1 \%$ & $2 \%$ & $3 \%$ & $4 \%$ & $5 \%$ & $10 \%$ & $20 \%$ & $30 \%$ & $40 \%$ & $50 \%$ \\
\hline \multirow{2}{*}{1189} & Median & $97,5 \%$ & $96,0 \%$ & $97,5 \%$ & $94,0 \%$ & $92,5 \%$ & $70,0 \%$ & $64,0 \%$ & $49,0 \%$ & $13,0 \%$ & $2,0 \%$ \\
\hline & $P 25-P 75$ & $96,5-98,3$ & $93,3-98,5$ & $92,3-99,0$ & $89,3-98,5$ & $84,3-98,0$ & $56,3-85,8$ & $49,8-78,5$ & $34,8-63,5$ & $0,0-38,0$ & $0,0-18,0$ \\
\hline \multirow{2}{*}{1190} & Median & $99,0 \%$ & $98,0 \%$ & $95,5 \%$ & $95,0 \%$ & $93,5 \%$ & $81,0 \%$ & $81,5 \%$ & $64,0 \%$ & $19,0 \%$ & $11,0 \%$ \\
\hline & $P 25-P 75$ & $98,8-99,3$ & $96,0-100,3$ & $92,0-99,3$ & $91,3-98,3$ & $90,0-96,0$ & $76,8-86,8$ & $72,0-86,8$ & $50,0-78,0$ & $0,0-47,5$ & $0,0-32,0$ \\
\hline \multirow{2}{*}{$10403 S$} & Median & $97,5 \%$ & $84,0 \%$ & $83,0 \%$ & $71,0 \%$ & $67,0 \%$ & $58,5 \%$ & $57,5 \%$ & $30,5 \%$ & $26,0 \%$ & $10,5 \%$ \\
\hline & $P 25-P 75$ & $90,3-101,0$ & $71,5-94,8$ & $63,8-98,8$ & $45,0-95,8$ & $42,3-93,0$ & $49,0-68,8$ & $43,5-73,5$ & $16,3-55,5$ & $18,0-41,8$ & $0,0-33,0$ \\
\hline \multirow{2}{*}{1044} & Median & $100,5 \%$ & $101,0 \%$ & $101,0 \%$ & $100,5 \%$ & $100,5 \%$ & $100,0 \%$ & $99,5 \%$ & $99,0 \%$ & $98,0 \%$ & $98,0 \%$ \\
\hline & P25-P75 & $99,8-101,3$ & $100,8-101,3$ & $100,5-101,3$ & $100,0-101,0$ & $99,8-101,0$ & $99,8-100,3$ & $97,8-101,0$ & $97,0-101,0$ & $94,8-101,0$ & $93,8-101,0$ \\
\hline
\end{tabular}

The supernatant of the 1044 mutant strain was used as a control sample not containing LLO. The percent given is that of living cells in the PI assay with different final supernatant concentrations of $1-50 \%$, following incubation for $30 \mathrm{~min}$ at $22^{\circ} \mathrm{C}$ (median values, $P 25-P 75, n=4$ ). The incubation medium contained $4 \%$ inactivated NCS.

TABLE 3: Survival of PBMC cells of healthy person treated with different concentrations of L. monocytogenes strain supernatants.

\begin{tabular}{|c|c|c|c|c|c|c|}
\hline \multicolumn{2}{|c|}{ Strain } & \multicolumn{5}{|c|}{ Supernatant concentrations } \\
\hline & & $10 \%$ & $20 \%$ & $30 \%$ & $40 \%$ & $50 \%$ \\
\hline \multirow{2}{*}{1044} & Median & $100,0 \%$ & $99,0 \%$ & $98,0 \%$ & $98,0 \%$ & $98,0 \%$ \\
\hline & $P 25-P 75$ & $96,5-100,5$ & $89,0-100,5$ & $90,5-99,5$ & $89,0-98,5$ & $86,5-98,0$ \\
\hline \multirow{2}{*}{$10403 S$} & Median & $78,0 \%$ & $47,0 \%$ & $8,0 \%$ & $8,0 \%$ & $7,0 \%$ \\
\hline & $P 25-P 75$ & $57,0-84,0$ & $29,0-55,5$ & $5,5-11,5$ & $6,5-10,0$ & $4,5-7,0$ \\
\hline \multirow{2}{*}{ MAC } & Median & $14,0 \%$ & $2,0 \%$ & $2,0 \%$ & $1,0 \%$ & $0,0 \%$ \\
\hline & $P 25-P 75$ & $9,5-33,5$ & $2,0-20,0$ & $1,0-4,0$ & $0,5-3,0$ & $0,0-3,0$ \\
\hline \multirow{2}{*}{1189} & Median & $76,0 \%$ & $65,0 \%$ & $49,0 \%$ & $18,0 \%$ & $11,0 \%$ \\
\hline & $P 25-P 75$ & $54,5-82,0$ & $35,0-79,0$ & $25,0-61,6$ & $9,5-20,0$ & $6,0-15,5$ \\
\hline \multirow{2}{*}{1190} & Median & $78,0 \%$ & $69,0 \%$ & $30,0 \%$ & $17,0 \%$ & $21,0 \%$ \\
\hline & P25-P75 & $60,5-83,5$ & $47,5-74,0$ & $17,0-38,0$ & $9,5-21,5$ & $10,5-23,5$ \\
\hline
\end{tabular}

The supernatant of the 1044 mutant strain was used as a control sample not containing LLO. The percent given is that of living cells in the PI assay with different final supernatant concentrations of $1-50 \%$, following incubation for $30 \mathrm{~min}$ at $22^{\circ} \mathrm{C}$ (median values, $P 25-P 75, n=4$ ). The incubation medium contained 4\% inactivated NCS. The activity of the MAC supernatant was significantly higher $(P<0.05)$ than those of the 10403 , 1189 , and 1190 strains. 
TABLE 4: Survival of PBMC cells of B-CLL patient treated with different concentrations of L. monocytogenes strain supernatants.

\begin{tabular}{|c|c|c|c|c|c|c|}
\hline \multicolumn{2}{|c|}{ Strain } & \multicolumn{5}{|c|}{ Supernatant concentrations } \\
\hline & & $10 \%$ & $20 \%$ & $30 \%$ & $40 \%$ & $50 \%$ \\
\hline \multirow{2}{*}{1044} & Median & $101,0 \%$ & $93,0 \%$ & $98,0 \%$ & $91,0 \%$ & $84,0 \%$ \\
\hline & $P 25-P 75$ & $100,5-102,0$ & $88,5-94,5$ & $95,5-98,0$ & $90,5-94,5$ & $80,0-92,0$ \\
\hline \multirow{2}{*}{$10403 S$} & Median & $53,0 \%$ & $27,0 \%$ & $12,0 \%$ & $3,0 \%$ & $9,0 \%$ \\
\hline & $P 25-P 75$ & $31,0-80,5$ & $16,0-63,5$ & $8,0-38,5$ & $2,0-17,0$ & $7,0-22,0$ \\
\hline \multirow{2}{*}{ MAC } & Median & $71,0 \%$ & $62,0 \%$ & $49,0 \%$ & $23,0 \%$ & $20,0 \%$ \\
\hline & $P 25-P 75$ & $36,5-83,5$ & $31,0-65,0$ & $24,5-51,0$ & $11,5-38,0$ & $10,0-25,0$ \\
\hline \multirow{2}{*}{1189} & Median & $49,0 \%$ & $23,0 \%$ & $14,0 \%$ & $11,0 \%$ & $19,0 \%$ \\
\hline & $P 25-P 75$ & $47,5-72,5$ & $20,5-61,5$ & $13,0-41,5$ & $8,0-30,0$ & $12,0-25,0$ \\
\hline \multirow{2}{*}{1190} & Median & $98,0 \%$ & $72,0 \%$ & $70,0 \%$ & $23,0 \%$ & $6,0 \%$ \\
\hline & $P 25-P 75$ & $65,5-98,0$ & $55,0-85,0$ & $48,0-85,0$ & $17,5-60,0$ & $5,5-7,0$ \\
\hline
\end{tabular}

Patients were not previously treated for leukemia. Cells were cultured in vitro for 6 days before the experiment. The percent given is that of living cells in the PI assay with different final supernatant concentrations of $10-50 \%$, following incubation for $30 \mathrm{~min}$ at $22^{\circ} \mathrm{C}$ (median values, $P 25-P 75, n=3$ ). The incubation medium contained $4 \%$ inactivated NCS. The activity of the MAC supernatant was not significantly different than that of supernatants of the 10403S, 1189 , and 1190 strains.

to the activities of other supernatants $(P<0.05)$ (Table 3); there was no statistically significant difference between supernatants of the strains 1189 and 1190 . The differences between activities of supernatants of strains 1189, 1190, and MAC were not observed with PBMC cells from BCLL patients. Overall, the responses of eukaryotic cells to bacteria supernatants depended on the products of the bacteria strain, the type of target eukaryotic cells, and the concentration of the supernatant in the experimental sample.

3.4. Activity of B. Subtilis Supernatants Against Peripheral Blood Mononuclear Cells or Jurkat Cells. The supernatants of hemolytically active samples from Bacillus subtilis with the hly gene coding for LLO (strains BR1-S, BR1-89, and BR1-90 grown with IPTG induction) exhibited cytotoxic activity in cytometric PI assays with the mononuclear cells of healthy subjects and with Jurkat cells (Tables 5 and 6). The bacterial culture medium contained IPTG to induce LLO expression and secretion. The tested concentration range of the original supernatants was $1-30 \%$ (Table 5) and there were two negative controls: BHI medium only and the supernatant of wild-type strain MB4 B. subtilis. The cytotoxic activity to PBMC targets was observed with supernatants of BR1-89 and BR1-S incubated with IPTG, starting from a concentration of $10 \%$ or $20 \%$, respectively. For BR1$90+$ IPTG, only the $30 \%$ supernatant concentration was effective (Table 6). Negative controls with BHI medium or supernatant of the MB4 strain were not cytotoxic under the examined conditions.

The cytotoxic activity of $B$. subtilis IPTG supernatants on Jurkat cells was concentration dependent $(P<0.0001)$. The supernatant of strain BR1-89 was more active; however, the difference from BR1-S was not statistically significant $(P>0.05)$. The BR1-90 supernatant was active with Jurkat cells within the concentration range $10-30 \%$. However, in this concentration range, the supernatant was inactive on PBMC from a healthy subject. The Jurkat cells appeared

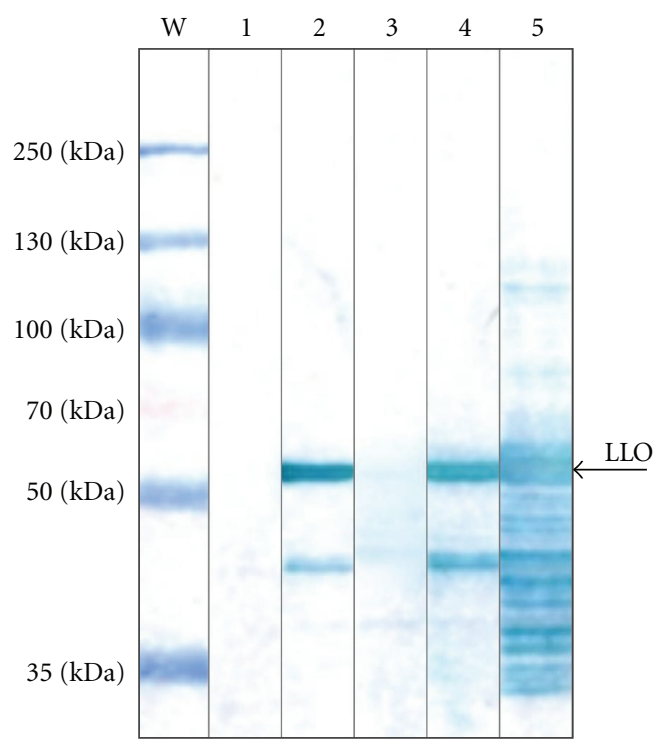

FIGURE 2: Western blot analysis of B. subtilis supernatants. Results of Western blot analysis of Bacillus subtilis supernatants with anti-LLO polyclonal antibody. 1: control wild-type strain MB4 supernatant, 2: BR1/S + IPTG, 3: BR1/S without IPTG, 4: BR1/89 + IPTG, 5: BR1/90 + IPTG. W: molecular weight marker. The LLO location is indicated.

more sensitive than PBMC to all tested supernatants $(P<$ 0.0001 ), and this difference was most pronounced with low concentrations of BR1-89+IPTG supernatant (Table 6).

Anti-LLO western blot analysis of supernatants produced by B. subtilis revealed a $58-\mathrm{kDa}$ protein (Figure 2). This LLO band was present in supernatants of strains BR1/S + IPTG, BR1/89 + IPTG, and BR1/90 + IPTG; however, it was not present in the supernatant of BR1/S without IPTG. The supernatants of BR1/90 contained several streaks reacting with anti-LLO antibody, probably fragments of LLO. Some 


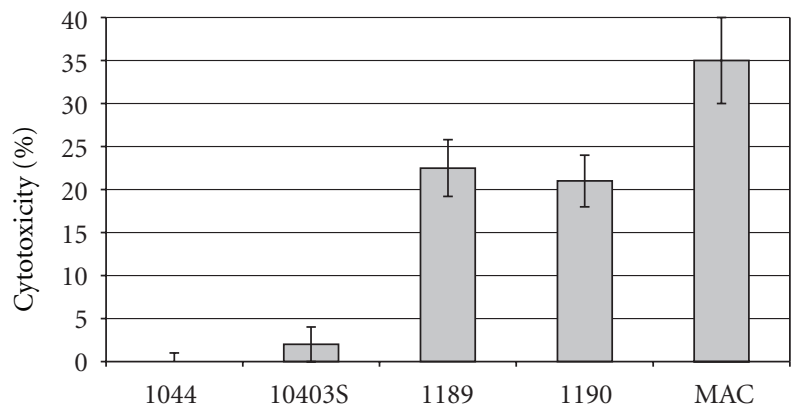

(a)

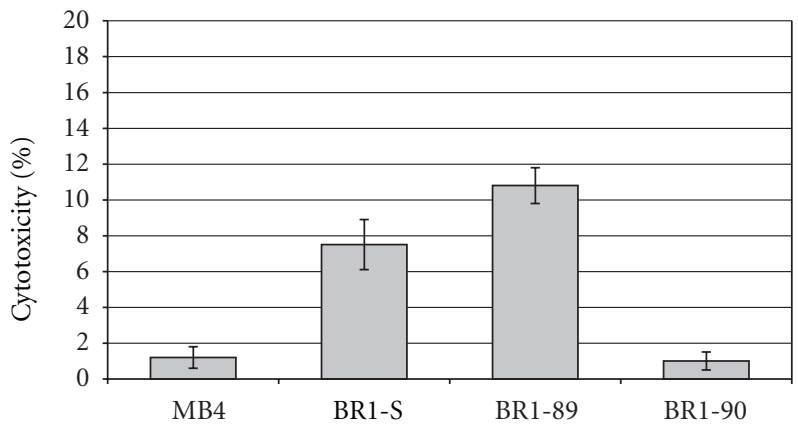

(b)

Figure 3: Cytotoxicity of L. monocytogenes (a) and B. subtilis (b) strains supernatants on Jurkat cells. Cytotoxicity of bacterial strain supernatants on the Jurkat cell line. Listeria monocytogenes (a) and Bacillus subtilis (b) supernatants were added to Jurkat cell cultures, and incubated for $1 \mathrm{~h}$ or $30 \mathrm{~min}$, respectively. Eukaryotic cells were stained for viability, and after 30 minutes were observed under fluorescent microscope. The DP-Soft program was used to determine the level of cytotoxicity induced by proteins produced by the bacterial strains. The bars indicate the means of the counts from three independent experiments, and the bars show the standard deviations.

TABLE 5: Survival of PBMC cells of healthy persons treated with different concentrations of B. subtilis supernatants.

\begin{tabular}{lccccccccc}
\hline & Strain & \multicolumn{7}{c}{ Supernatant concentrations } \\
& & $1 \%$ & $2 \%$ & $3 \%$ & $4 \%$ & $5 \%$ & $10 \%$ & $20 \%$ & $30 \%$ \\
\hline \multirow{2}{*}{ MB4 } & Median & $101,3 \%$ & $101,3 \%$ & $100,0 \%$ & $100,0 \%$ & $100,0 \%$ & $97,4 \%$ & $96,1 \%$ & $94,7 \%$ \\
& P25-P75 & $100,7-102,0$ & $100,7-101,3$ & $98,0-101,3$ & $100,0-100,7$ & $100,0-100,7$ & $96,7-100,0$ & $94,7-97,4$ & $94,1-95,4$ \\
\hline \multirow{2}{*}{ BHI medium } & Median & $100,0 \%$ & $98,7 \%$ & $98,7 \%$ & $100,0 \%$ & $100,0 \%$ & $98,7 \%$ & $96,1 \%$ & $96,1 \%$ \\
& P25-P75 & $99,3-101,3$ & $98,0-101,3$ & $98,0-100,7$ & $98,7-100,7$ & $99,3-100,0$ & $96,7-100,0$ & $96,1-97,4$ & $94,7-96,7$ \\
\hline \multirow{2}{*}{ BR1-90 } & Median & $98,7 \%$ & $100,0 \%$ & $103,9 \%$ & $100,0 \%$ & $100,0 \%$ & $98,7 \%$ & $97,4 \%$ & $90,8 \%$ \\
& P25-P75 & $97,4-100,7$ & $99,3-100,7$ & $100,7-104,6$ & $99,3-101,3$ & $95,4-100,0$ & $97,4-100,0$ & $96,7-97,4$ & $90,1-92,8$ \\
\hline \multirow{2}{*}{ BR1-89 } & Median & $98,7 \%$ & $101,3 \%$ & $100,0 \%$ & $98,7 \%$ & $98,7 \%$ & $92,1 \%$ & $47,4 \%$ & $7,9 \%$ \\
& P25-P75 & $98,7-100,0$ & $95,4-102,0$ & $98,7-100,7$ & $98,0-99,3$ & $98,0-98,7$ & $92,1-92,8$ & $35,5-67,1$ & $5,3-30,9$ \\
\hline \multirow{2}{*}{ BR1-S } & Median & $98,7 \%$ & $97,4 \%$ & $100,0 \%$ & $97,4 \%$ & $94,7 \%$ & $96,1 \%$ & $75,0 \%$ & $35,5 \%$ \\
& P25-P75 & $98,7-100,7$ & $97,4-100,0$ & $100,0-100,7$ & $90,1-100,0$ & $93,4-98,7$ & $95,4-100,0$ & $57,2-85,5$ & $23,0-55,3$ \\
\hline
\end{tabular}

The BHI medium and the supernatant of the MB4 wild-type strain were used as a control sample not containing LLO. The percent given is of living cells in the PI assay for different final IPTG-treated supernatant concentrations of $1-30 \%$, when Jurkat cells were incubated for 30 min at $22^{\circ} \mathrm{C}$ (median values, $P 25-$ $P 75, n=4)$. The incubation medium contained $4 \%$ inactivated NCS. The activities of BR1-89, BR1-90, and BR1-S were not significantly different from each other. Their activities were all significantly higher compared with that of the control supernatant.

TABLE 6: Survival of Jurkat cells treated with different concentrations of B. subtilis supernatants.

\begin{tabular}{lccccccccc}
\hline & Strain & \multicolumn{7}{c}{ Supernatant concentrations } \\
& & $1 \%$ & $2 \%$ & $3 \%$ & $4 \%$ & $5 \%$ & $10 \%$ & $20 \%$ & $30 \%$ \\
\hline \multirow{2}{*}{ MB4 } & Median & $101,4 \%$ & $109,3 \%$ & $108,6 \%$ & $109,3 \%$ & $111,4 \%$ & $111,4 \%$ & $119,3 \%$ & $121,4 \%$ \\
& $P 25-P 75$ & $96,4-106,4$ & $108,2-110,0$ & $106,8-112,5$ & $104,3-113,6$ & $109,6-115,0$ & $109,6-114,3$ & $117,9-120,4$ & $118,9-123,6$ \\
\hline \multirow{2}{*}{ BHI medium } & Median & $105,0 \%$ & $104,3 \%$ & $100,7 \%$ & $105,0 \%$ & $101,4 \%$ & $100,7 \%$ & $100,7 \%$ & $96,4 \%$ \\
& P25-P75 & $104,3-106,8$ & $100,7-106,4$ & $98,9-102,1$ & $102,9-107,5$ & $100,7-102,5$ & $99,6-102,5$ & $99,3-101,4$ & $94,6-97,5$ \\
\hline \multirow{2}{*}{ BR1-90 } & Median & $105,6 \%$ & $104,9 \%$ & $102,8 \%$ & $98,6 \%$ & $93,7 \%$ & $73,2 \%$ & $40,8 \%$ & $8,5 \%$ \\
& P25-P75 & $102,-109,2$ & $101,4-108,5$ & $96,8-107,0$ & $90,1-104,2$ & $83,1-102,5$ & $62,0-89,4$ & $34,5-44,0$ & $8,1-8,5$ \\
\hline \multirow{2}{*}{ BR1-89 } & Median & $98,1 \%$ & $93,1 \%$ & $83,8 \%$ & $68,1 \%$ & $32,5 \%$ & $4,4 \%$ & $0,0 \%$ & $0,0 \%$ \\
& P25-P75 & $94,1-101,6$ & $91,3-95,6$ & $78,1-88,8$ & $64,4-68,8$ & $23,4-38,4$ & $3,4-5,3$ & $0,0-0,3$ & $0,0-0,3$ \\
\hline \multirow{2}{*}{ BR1-S } & Median & $100,0 \%$ & $98,7 \%$ & $96,8 \%$ & $94,3 \%$ & $78,5 \%$ & $25,9 \%$ & $1,9 \%$ & $1,3 \%$ \\
& P25-P75 & $99,1-101,3$ & $97,2-100,6$ & $96,2-98,1$ & $88,0-96,8$ & $76,3-85,4$ & $17,4-35,1$ & $1,3-2,5$ & $0,9-1,6$ \\
\hline
\end{tabular}

The BHI medium and the supernatant of the MB4 wild-type strain were used as a control sample not containing LLO. The percent given is of living cells in the PI assay for different final supernatant concentrations of $1-30 \%$, when PBMC were incubated for 30 min at $22^{\circ} \mathrm{C}$ (median values, $P 25-P 75, n=3$ ). The incubation medium contained 4\% inactivated NCS. The activity of BR1-90 was not significantly different from that of the control supernatant of the MB4 strain; however, supernatant activities of BR1-89 and BR1-S were significantly higher. 
fragments were complexed to events of MW $>58 \mathrm{kDa}$. The supernatant was not hemolytic.

In summary, the supernatants of B. subtilis BR1-S and BR1-89 strains were cytotoxic to normal PBMC and to Jurkat T-leukemia cells. The Jurkat cells were more sensitive to the supernatantsthan PBMC of a healthy person. The supernatant of the BR1-90 strain was not hemolytic and not cytotoxic to PBMC within the range of $1-30 \%$, or to Jurkat cells in the range of $1-5 \%$; however, it was cytotoxic to Jurkat cells within the range of $10-30 \%$, suggesting that LLO is selectively effective against T-leukemia cells.

\section{Discussion}

Listeria monocytogenes is commonly used in biomedical research, due to its unique cell translocation cycle and the extensive available knowledge of its biology. It is most often used as a vector for inducing cell-mediated immunity. In this experiment, we present another approach-the direct use of the cytotoxic potential of listerial toxins. Such activity could be used as an alternative to traditional chemotherapy. We used L. monocytogenes strains synthesizing LLO and mutant strains producing variants of this toxin, along with engineered $B$. subtilis strains harboring a wild-type or mutated hlyA gene. Although optimal activity of LLO is displayed in acidic conditions [23], we tested the activity of bacterial supernatants at neutral $\mathrm{pH}$. In such conditions, both the wild-type strain and the mutant $L$. monocytogenes strains used in this study have previously shown no detectable hemolytic activity [24]. Therefore, we enhanced the toxin productions of these strains using the well-known "active charcoal phenomenon" [25] that maximizes PrfAdependant gene expression. We also used mutant strains containing prfA* mutations [26] that lead to overproduction of pathogenicity determinants, such as the MAC strain. Both approaches proved effective, since we recorded significant cytolytic activity directed against leukemia cells in vitro, comparable to the activity of the supernatants in optimal conditions ( $\mathrm{pH} 5.6$ with a reducing agent) isolated from the same strains but grown under standard conditions [24]. The experimental model used in the present study was valuable for in vitro hemolytic and cytotoxic assays of bacterial products containing LLO on human normal and leukemia leukocytes. The tested bacteria strains containing the hly gene secreted listeriolysin $\mathrm{O}$ into the culture medium (supernatant). These supernatants exhibited hemolytic or cytotoxic activities as compared with supernatants of the control samples not secreting listeriolysin $\mathrm{O}$. The presence of LLO in active supernatants was confirmed by western blot analysis. The results of hemolytic and cytoxicity tests cannot be directly compared to each other; however, the results were similar, as supernatants of Listeria and Bacillus strains not secreting LLO were negative at a broad range of concentrations, and the supernatants of LLO-secreting bacteria were active. Differences in cytotoxic activity between supernatants of different tested strains of Listeria or Bacillus were not significant, except for the greater hemolytic activities of supernatants of Listeria strain MAC and Bacillus strain
BR1-89. The supernatants appeared to be cytotoxic to human leukocytes in a concentration-dependent way.

The employment of $B$. subtilis strains producing LLO is an attractive idea because it enables the study of this toxin in isolation from other pathogenesis factors [15]. Such practice also potentiates the practical use of such agents due to the much greater safety of $B$. subtilis-derived proteins compared to working with the original pathogenic host. In the present study, we recorded significant differences between Bacillus strains expressing different LLO variants, which were not observed between L. monocytogenes strains expressing different LLO variants, here or in our previous [24] study. The main reason for the observed similar activities of $L$. monocytogenes supernatants containing different LLO variants is most likely the high phospholipase activity obscuring subtle changes in LLO activity [7].The cytotoxic activity may depend on variations of cell membrane cholesterol present in different target cells $[27,28]$. It has been suggested that domains of the cell membrane enriched in cholesterol and sphingolipids are responsible for the formation of lipid rafts involved in the regulation of several biological processes $[29,30]$. The cell membrane cholesterol binding of LLO has been demonstrated for the mouse monocyte-macrophage cell line J774 and for erythrocyte membranes [27]. In our experiment, the observed supernatant cytotoxicity was different toward the various target cell populations. The most sensitive were the human T-leukemia Jurkat cells, while normal peripheral blood PBMC and PBMC of B-cell chronic lymphocytic leukemia patients were more refractory.

This finding that LLO activity is apparently directed more to $\mathrm{T}$ cells than B cells may have some medical consequences, especially in T-cell lymphoma. Angioimmunoblastic T-cell lymphoma is a distinct peripheral T-cell lymphoma entity with peculiar clinical and pathological features [31]. These patients typically have a poor outcome when treated with conventional chemotherapy regimens, and future efforts should be directed at evaluating the use of LLO activity to create promising innovative therapies.

\section{Acknowledgments}

This work was supported by the European Union structural funds, the Innovative Economy Operational Program POIG.01.01.02-00-109/09-00. The study was partly supported by Grant N N401 015936 obtained from the Polish Ministry of Science and Higher Education and Grant CMKP 2008/2009. The authors thank Dr. Krystyna Stepien from the Diagnostic Laboratory CSK MSWiA for cholesterol assays.

\section{References}

[1] L. R. Zacharski and V. P. Sukhatme, "Coley's toxin revisited: immunotherapy or plasminogen activator therapy of cancer?" Journal of Thrombosis and Haemostasis, vol. 3, no. 3, pp. 424427, 2005.

[2] S. A. Hoption Cann, J. P. van Netten, and C. van Netten, "Dr William Coley and tumour regression: a place in history or in the future," Postgraduate Medical Journal, vol. 79, no. 938, pp. 672-680, 2003. 
[3] J. E. Alouf and M. R. Popoff, The Comprehensive Sourcebook of Bacterial Protein Toxins, Academic Press, London, UK, 2005.

[4] J. E. Alouf, S. J. Billington, and B. H. Jost, "Repertoire and general features of the family of cholesterol-dependent cytolysins," in The Comprehensive Sourcebook of Bacterial Protein Toxins, J. E. Alouf and M. R. Popoff, Eds., pp. 643-658, Academic Press, London, UK, 2005.

[5] D. A. Portnoy, P. S. Jacks, and D. J. Hinrichs, "Role of hemolysin for the intracellular growth of Listeria monocytogenes," The Journal of Experimental Medicine, vol. 167, no. 4, pp. 14591471, 1988.

[6] J. A. Vazquez-Boland, R. Stachowiak, L. Lacharme, and M. Scortti, "Listeriolysin," in The Comprehensive Sourcebook of Bacterial Protein Toxins, J. E. Alouf and M. R. Popoff, Eds., pp. 700-716, Academic Press, London, UK, 2005.

[7] R. Stachowiak and J. Bielecki, "Contribution of hemolysin and phospholipase activity to cytolytic properties and viability of Listeria monocytogenes," Acta Microbiologica Polonica, vol. 50, no. 3-4, pp. 243-250, 2001.

[8] C. Alberti-Segui, K. R. Goeden, and D. E. Higgins, "Differential function of Listeria monocytogenes listeriolysin $\mathrm{O}$ and phospholipases $\mathrm{C}$ in vacuolar dissolution following cell-to-cell spread," Cellular Microbiology, vol. 9, no. 1, pp. 179-195, 2007.

[9] G. A. Smith, H. Marquis, S. Jones, N. C. Johnston, D. A. Portnoy, and H. Goldfine, "The two distinct phospholipases C of Listeria monocytogenes have overlapping roles in escape from a vacuole and cell-to-cell spread," Infection and Immunity, vol. 63, no. 11, pp. 4231-4237, 1995.

[10] L. L. Fu, Z. R. Xu, W. F. Li, J. B. Shuai, P. Lu, and C. X. Hu, "Protein secretion pathways in Bacillus subtilis: implication for optimization of heterologous protein secretion," Biotechnology Advances, vol. 25, no. 1, pp. 1-12, 2007.

[11] R. Nijland and O. P. Kuipers, "Optimization of protein secretion by Bacillus subtilis," Recent Patents on Biotechnology, vol. 2, no. 2, pp. 79-87, 2008.

[12] M. Schallmey, A. Singh, and O. P. Ward, "Developments in the use of Bacillus species for industrial production," Canadian Journal of Microbiology, vol. 50, no. 1, pp. 1-17, 2004.

[13] M. Desvaux and M. Hébraud, "The protein secretion systems in Listeria: inside out bacterial virulence," FEMS Microbiology Reviews, vol. 30, no. 5, pp. 774-805, 2006.

[14] J. Bielecki, P. Youngman, P. Connelly, and D. A. Portnoy, "Bacillus subtilis expressing a haemolysin gene from Listeria monocytogenes can grow in mammalian cells," Nature, vol. 345, no. 6271, pp. 175-176, 1990.

[15] J. Kawiak, R. Stachowiak, M. Lyzniak, J. Bielecki, and L. Granicka, "The use of hollow fiber membranes combined with cytometry in analysis of bacteriological samples," Methods in Cell Biology, vol. 102, pp. 411-429, 2011.

[16] R. Stachowiak, L. H. Granicka, J. Wisniewski, M. Lyzniak, J. Kawiak, and J. Bielecki, "Cytotoxicity of listeriolysin O produced by membrane-encapsulated Bacillus subtilis on leukemia cells," Journal of Microbiology and Biotechnology, vol. 21, no. 11, pp. 1193-1198, 2011.

[17] D. K. Bishop and D. J. Hinrichs, "Adoptive transfer of immunity to Listeria monocytogenes: the influence of in vitro stimulation on lymphocyte subset requirements," Journal of Immunology, vol. 139, no. 6, pp. 2005-2009, 1987.

[18] J. Bielecki, "Association of listeriolysin O with the cell surface of Listeria monocytogenes," Acta Microbiologica Polonica, vol. 43, no. 3-4, pp. 279-289, 1994.

[19] A. Camilli, C. R. Paynton, and D. A. Portnoy, "Intracellular methicillin selection of Listeria monocytogenes mutants unable to replicate in a macrophage cell line," Proceedings of the
National Academy of Sciences of the United States of America, vol. 86 , no. 14 , pp. 5522-5526, 1989.

[20] K. Miki and G. B. Mackaness, "The passive transfer of acquired resistance to Listeria monocytogenes," The Journal of Experimental Medicine, vol. 120, pp. 93-103, 1964.

[21] D. A. Portnoy, R. K. Tweten, M. Kehoe, and J. Bielecki, "Capacity of listeriolysin $\mathrm{O}$, streptolysin $\mathrm{O}$, and perfringolysin $\mathrm{O}$ to mediate growth of Bacillus subtilis within mammalian cells," Infection and Immunity, vol. 60, no. 7, pp. 2710-2717, 1992.

[22] P. Zuber and R. Losick, "Role of AbrB in Spo0A- and Spo0Bdependent utilization of a sporulation promoter in Bacillus subtilis," Journal of Bacteriology, vol. 169, no. 5, pp. 2223-2230, 1987.

[23] C. Geoffroy, J. L. Gaillard, J. E. Alouf, and P. Berche, "Purification, characterization, and toxicity of the sulfhydrylactivated hemolysin listeriolysin $\mathrm{O}$ from Listeria monocytogenes," Infection and Immunity, vol. 55, no. 7, pp. 1641-1646, 1987.

[24] R. Stachowiak, J. Wiśniewski, O. Osińska, and J. Bielecki, "Contribution of cysteine residue to the properties of Listeria monocytogenes listeriolysin O," Canadian Journal of Microbiology, vol. 55, no. 10, pp. 1153-1159, 2009.

[25] S. Ermolaeva, S. Novella, Y. Vega, M. T. Ripio, M. Scortti, and J. A. Vázquez-Boland, "Negative control of Listeria monocytogenes virulence genes by a diffusible autorepressor," Molecular Microbiology, vol. 52, no. 2, pp. 601-611, 2004.

[26] M. T. Ripio, G. Domínguez-Bernal, M. Lara, M. Suárez, and J. A. Vázquez-Boland, "A Gly145Ser substitution in the transcriptional activator PrfA causes constitutive overexpression of virulence factors in Listeria monocytogenes," Journal of Bacteriology, vol. 179, no. 5, pp. 1533-1540, 1997.

[27] A. Bavdek, N. O. Gekara, D. Priselac et al., "Sterol and pH interdependence in the binding, oligomerization, and pore formation of listeriolysin O," Biochemistry, vol. 46, no. 14, pp. 4425-4437, 2007.

[28] K. M. Habegger, N. J. Hoffman, C. M. Ridenour, J. T. Brozinick, and J. S. Elmendorf, "AMPK enhances insulinstimulated GLUT4 regulation via lowering membrane cholesterol," Endocrinology, vol. 153, no. 5, pp. 2130-2141, 2012.

[29] A. D. Goddard and A. Watts, "Regulation of G protein-coupled receptors by palmitoylation and cholesterol," BMC Biology, vol. 10, article 27, 2012.

[30] N. Fedoryszak-Kuska, M. Panasiewicz, and T. Pacuszka, "Lipid rafts-remarks and doubts," Advances in Cell Biology, vol. 38, pp. 313-332, 2011.

[31] L. de Leval, C. Gisselbrecht, and P. Gaulard, "Advances in the understanding and management of angioimmunoblastic Tcell lymphoma," British Journal of Haematology, vol. 148, no. 5, pp. 673-689, 2010. 

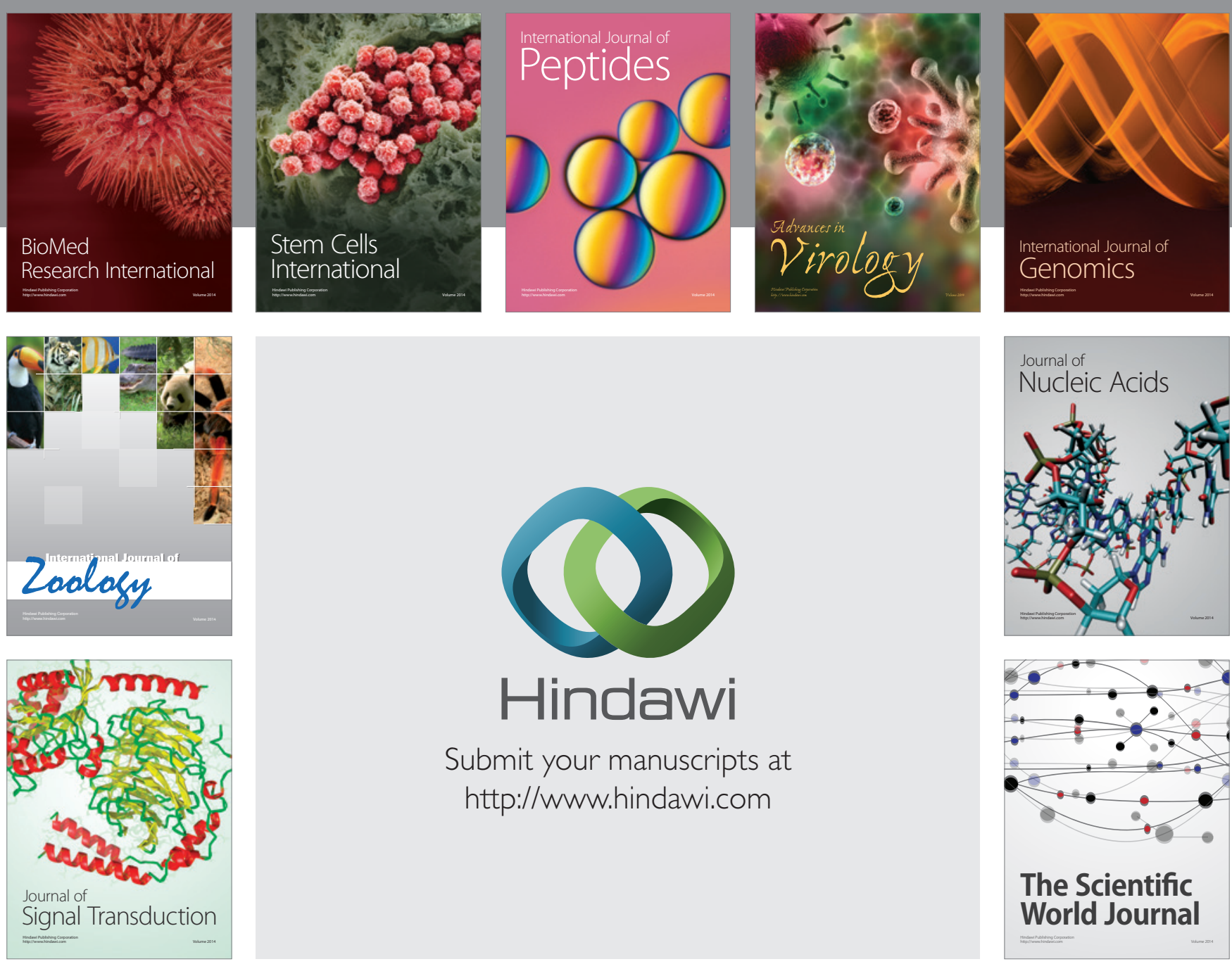

Submit your manuscripts at

http://www.hindawi.com
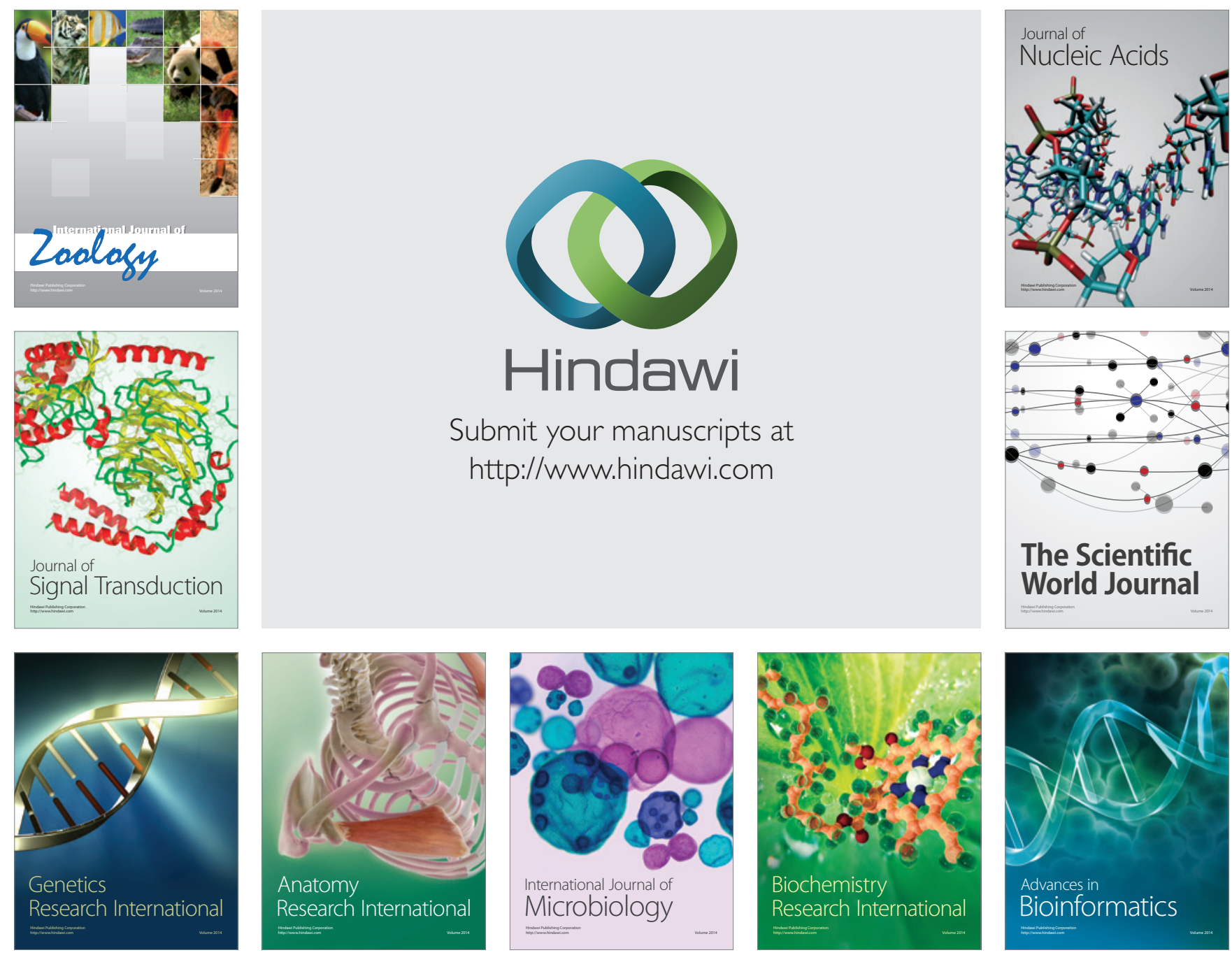

The Scientific World Journal
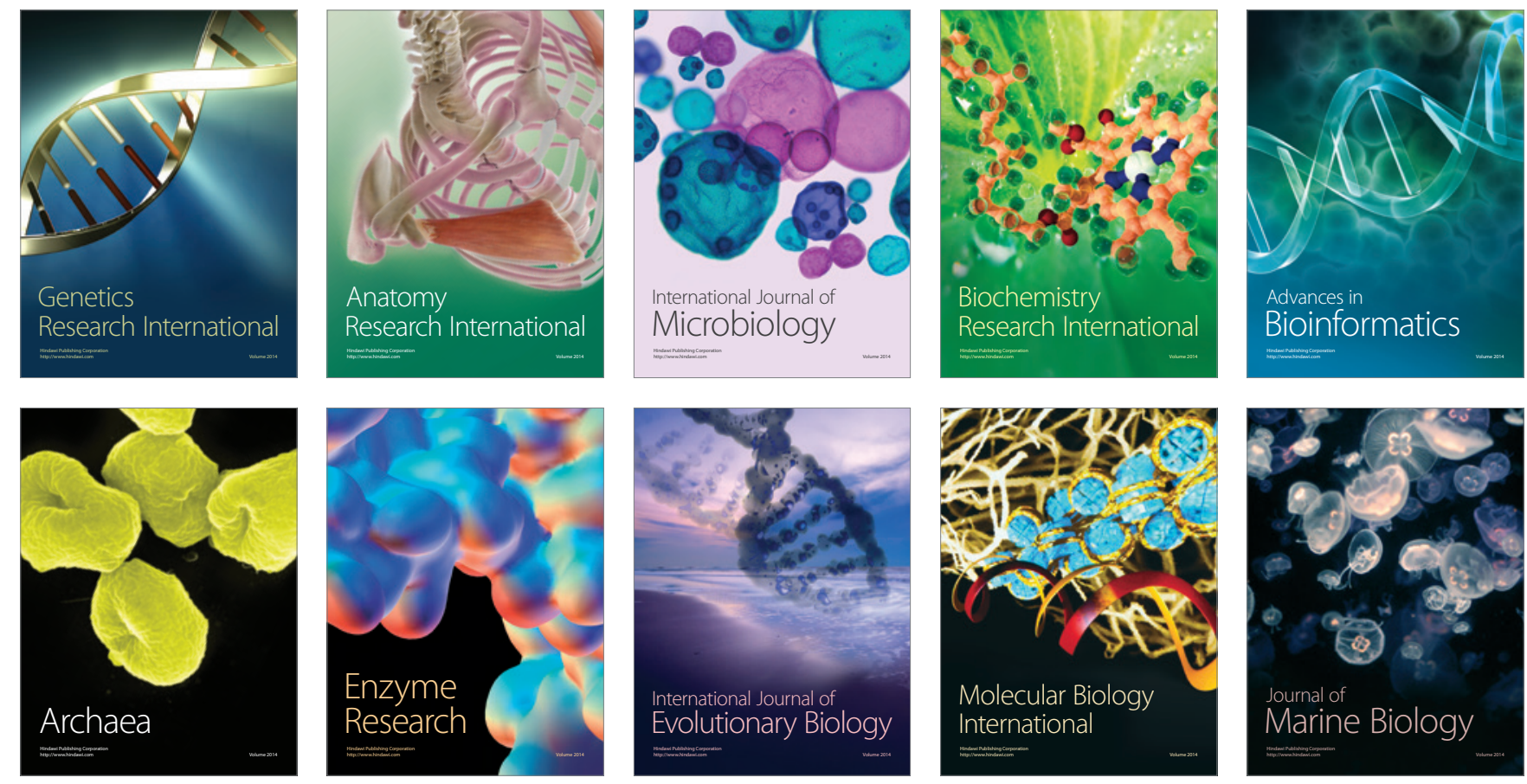